\title{
Repeated use of mechanical restraints in patients admitted to an acute psychiatric ward
}

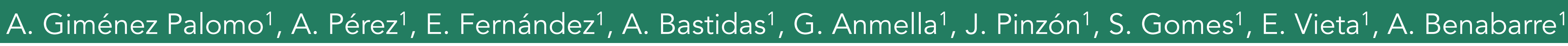

${ }^{1}$ Department of Psychiatry. Institut Clínic de Neurociències. Hospital Clínic de Barcelona. Barcelona, Spain.

email address: agimenezp@clinic.cat

\section{INTRODUCTION}

The use of coercive measures in psychiatric practice has been a continued matter of concern, which has raised the necessity of learning different skills, such as verbal de-escalation and conflict behavior prevention, in order to improve therapeutic outcomes. In this context, a training plan called 'Zero Containment' was implemented in Hospital Clínic of Barcelona from 2016. This consisted in updating the knowledge and training of health care personnel nurses, nursing assistants and health aides.

\section{OBJECTIVES}

To evaluate the relationship between the repeated use of mechanical restraints and previous admissions to a psychiatric ward and also between multiple use of mechanical restraints and the patients' diagnoses.

\section{METHODS}

An observational retrospective analysis of mechanical restraints in our acute psychiatric ward was done from registered data from 2012 to 2016, containing a sample of 193 patients. The statistical method used was Chi Square.

\section{RESULTS}

Our results show a reduction in the number of mechanical restraints after the implementation of our training plan. $35.4 \%$ of patients who required mechanical restriction were contained a second time. There were no significant differences between the use of mechanical restraints repeatedly and previous psychiatric admissions $(p=0.42) .88 .6 \%$ of patients had psychotic or major affective disorders. When comparing the repeated use of mechanical restraints according to their diagnoses, no significant differences were found between psychotic or major affective patients and patients with other diagnoses $(p=0.99)$.
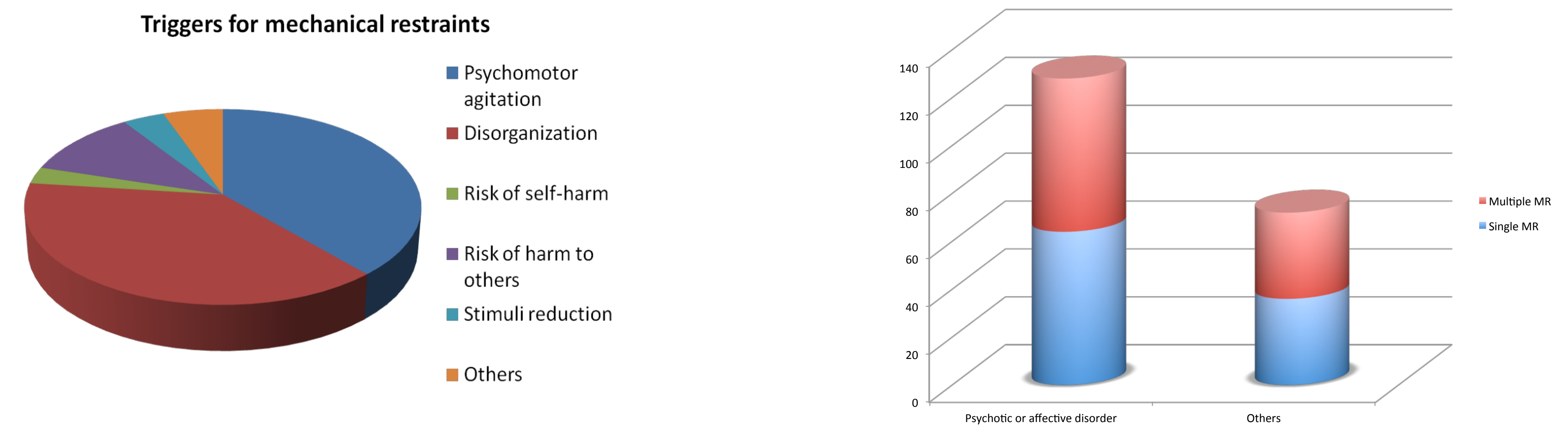

\section{CONCLUSIONS}

In conclusion, more than one third of patients who required a mechanical restriction during their admission presented further episodes that ended up with new episodes of mechanical restrictions. These data emphasize the importance of working with these patients towards the prevention of new episodes by using verbal de-escalation.

\section{REFERENCES}

[1] Zeller, S.L., Citrome, L., 2016. Managing Agitation Associated with Schizophrenia and Bipolar Disorder in the Emergency Setting. West J Emerg Med. 17(2): 165-172. [2] Richardson, M., Brennan, G., James, K., Lavelle, M., Renwick, L., Stewart, D., Bowers, L., 2015. Describing the precursors to and management of medication nonadherence on acute psychiatric wards. Gen Hosp Psychiatry 37(6):606-12. 